\title{
Producing Reusable Web-Based Multimedia Presentations
}

\section{Freimut Bodendorf and Manfred Schertler Department of Information Systems, University of Erlangen-Nuremberg, Germany}

freimut.bodendorf@wiso.uni-erlangen.de manfred.schertler@wiso.uni-erlangen.de

\author{
Eli Cohen \\ Informing Science \\ Institute, USA and \\ Kozminski School of \\ Entrepreneurship, \\ Warsaw, Poland \\ EliCohen@ \\ informingscience.org
}

\begin{abstract}
This paper describes the process and particular support tools for authoring reusable web-based multimedia presentations. These tools enable authors to record and encode streams of audio and video, and to integrate into them additional multimedia material, such as PowerPoint slides. The paper also describes the W3C synchronized multimedia integration language (SMIL) standard that enables the interlinking of audio/video with additional material. Finally, the paper introduces an easy-to-use authoring tool, developed and used by the author's faculty to produce multimedia information packages, and their corresponding user interface.
\end{abstract}

\section{Introduction}

A review of any number of recent journals makes it clear that traditional universities increasingly are placing course material and even entire courses on the Web. Different universities use different approaches, often differing in both how they present courses to students and how students are to learn using these environments. Regardless of their differences, the key factor for change is the university's recognition that time and location flexibility is essential for the success of any distance education program. State of the art data networks with scaleable bandwidth for different multimedia distribution scenarios are already in use for synchronous teleteaching, the transmission of lectures and seminars (Klein, 1999). In addition, universities are increasingly examining the potential for asynchronous teleteaching, the production of presentation material for ondemand use.

Our research focuses on an instance of asynchronous teleteaching that we call "Lecture of Demand”, LoD. We developed a LoD toolset for producing reusable web-based multimedia presentations that records online lectures for future replay and also enables editing of the re-

Material published as part of this journal, either on-line or in print, is copyrighted by the publisher of the Informing Science Journal. Permission to make digital or paper copy of part or all of these works for personal or classroom use is granted without fee provided that the copies are not made or distributed for profit or commercial advantage AND that copies 1) bear this notice in full and 2) give the full citation on the first page. It is permissible to abstract these works so long as credit is given. To copy in all other cases or to republish or to post on a server or to redistribute to lists requires specific permission and payment of a fee. Contact Publisher@ijklo.org to request redistribution permission. cording, and the adding of additional material and links, including slides, annotations, diagrams, animations, and such.

The idea behind reusable web-based multimedia presentations is simple to grasp, but perhaps not so simple to implement. The idea involves two components. The first component is developing and recording the best 
possible exposition on each topic taught. Of course, there are many such "best" expositions as each course taught is aimed at students with a given set of prerequisite knowledge and skills, and this set is dependent upon the curriculum. That is, each exposition should build upon prior knowledge (and not require skills not yet present in the student). For example, there is not one single best exposition or book on the topic "Introduction to Database" because the topic is different for students of computer science compared with business students, and undergraduates compared with graduate students.

The exposition developed in this paper is based on well-designed/edited lectures, supplemented with multimedia clarifications, exhibits, and expositions, as well as links for learning missing prerequisite knowledge and/or exploring the topic in greater depth. Developing these expositions requires more initial effort by our staff; in return, it provides the opportunity for reuse.

The second component of web-based multimedia presentations is presenting the developed exposition at the most opportune time. Once created, we can offer the web-based multimedia presentations to our students repeatedly. For example, a given student may review a given module several times. Likewise, students lacking the required/prerequisite knowledge are directed to review a presentation on this knowledge area that might have been developed for the prerequisite course. The course designer's task is sequencing the reusable web-based multimedia presentations for students.

This paper illustrates the process of producing a LoD. In brief, the paper describes a technical solution based on creating an XML application that follows the W3C recommendation called Synchronized Multimedia Integration Language (SMIL) when creating source material. SMILcompliant source files can be displayed using software readily available to students and others without charge. This approach also enables the university to provide the materials to students via less-expensive low bandwidth lines.

Figure 1 shows a system overview of the production process and the technology used for LoD.

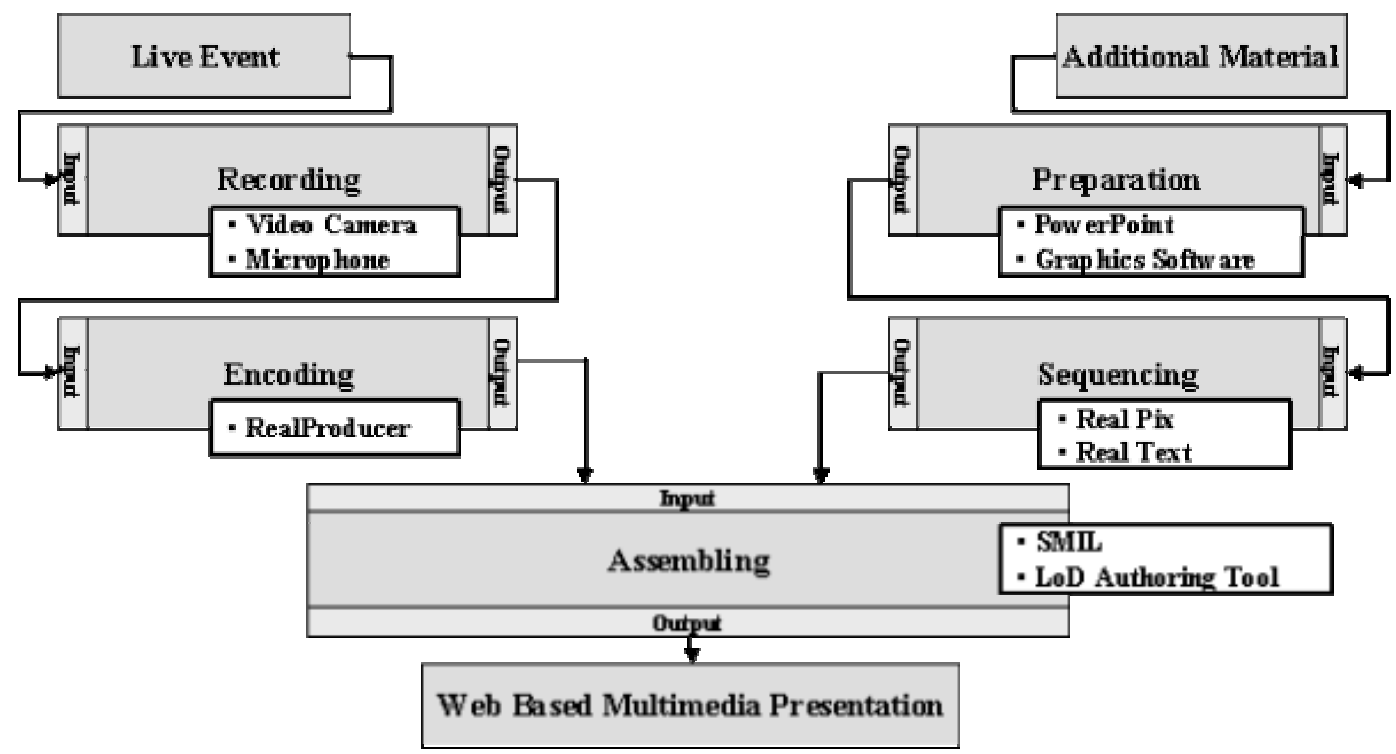

Figure 1: System Overview

\section{Other Approaches to Provide Lectures on Demand}

The LoD toolset evolved, in part due to the limitations of other systems to provide the solutions we needed. This section describes four systems that provide multimedia information presentations 
over the Internet. They are as follows: D-Lecture, LECTURNITY, Microsoft Producer, and IControl. This section summarizes the results of research conducted in our department and previously published (Knauer, 2004).

\section{D-Lecture}

D-Lecture is the result of a collaborative project by the University of Bremen and the Bremen Briteline GmbH (http://www.d-lecture.de/projekt/projektbeschreibung.htm). Its objective is to provide lecture and presentation content together with digital additional material over the Internet. To view lectures encoded and delivered by D-Lecture, users need only a web browser and a browser plug-in provided free by Real Media. The main screen of D-Lecture is divided into four areas: video, slides, lecture structure, and a combined area with navigation elements, web links, and annotations. Figure 2 shows the user interface of D-Lecture.

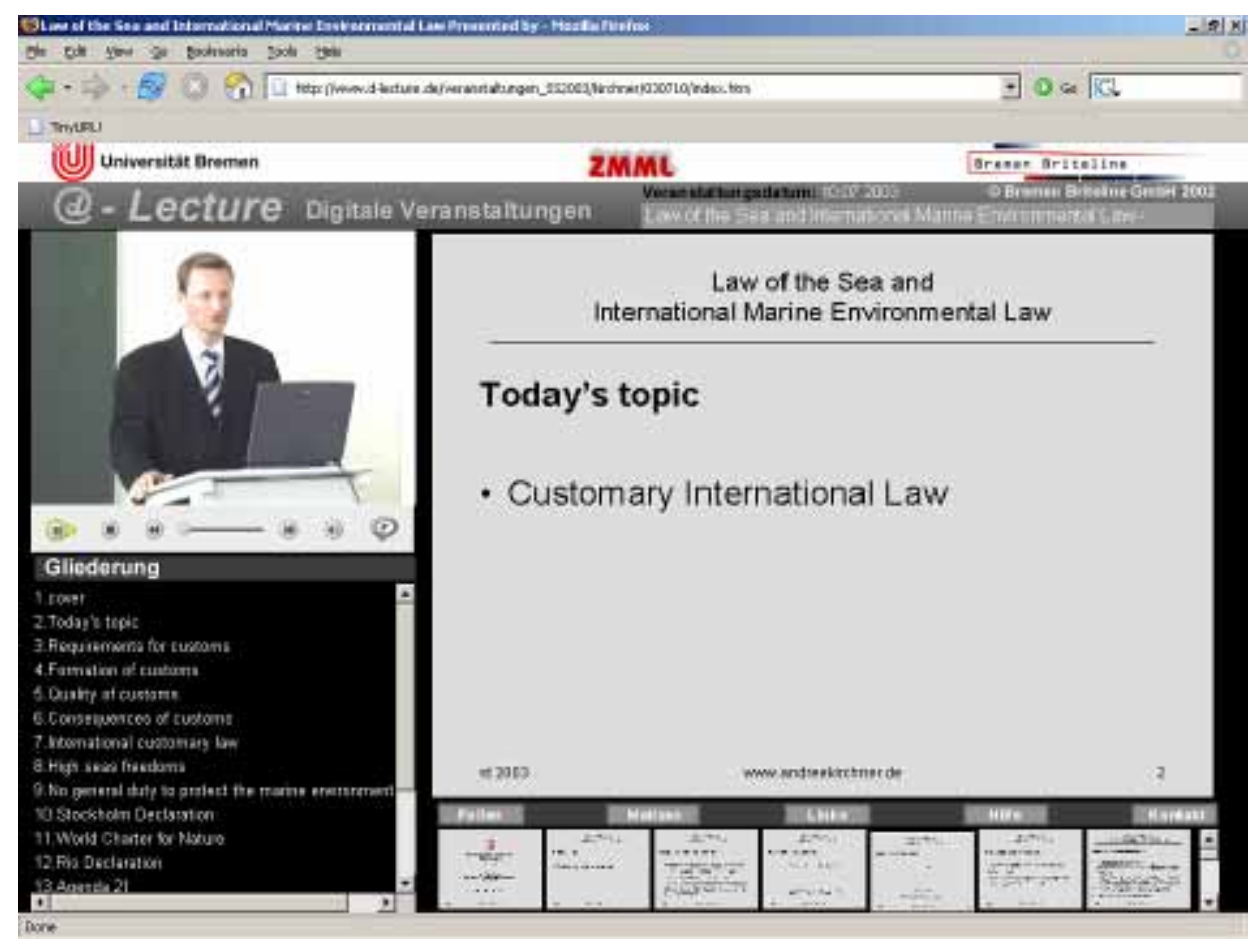

Figure 2: Screenshot D-Lecture User Interface

The video stream includes time stamps to enable it and additional material to stay synchronized The Real Media plug-in interprets these timestamps and assigns the browser to show the appropriate slide at the right time.

However, D-Lectures are difficult to edit or otherwise modify. Even adding or removing a slide requires a great deal of work.

D-Lecture supports Real Media videos, JPEG, and GIF graphics and is prepared to integrate MPEG and Quicktime videos as well.

\section{LECTURNITY}

LECTURNITY (http://www.im-c.de/lecturnity/) is real-time screen recording software developed and distributed by imc AG. This authoring system combines the recording of text, audio, video, screen images, and annotations in a single application. 
Starting point of a LECTURNITY authoring process is a PowerPoint file. The software assists the author during the export procedure. The converted PowerPoint slides and screen recordings are the basic types of additional material provided by the system. Videos and screen capture sequences are recorded "on-the-fly" with the system automatically recording timestamps to signal when to display slides. To view the recorded lectures, LECTURNITY recommends using its own player, but any standard browser will play the recorded material. LECTURNITY supports Real Media and Windows Media videos, Flash presentations, GIF, and PNG graphics.

LECTURNITY includes the recording of voice, slides, and such, but does not provide support for giving quizzes or assignments. Figure 3 shows a screenshot of the LECTURNIY player.

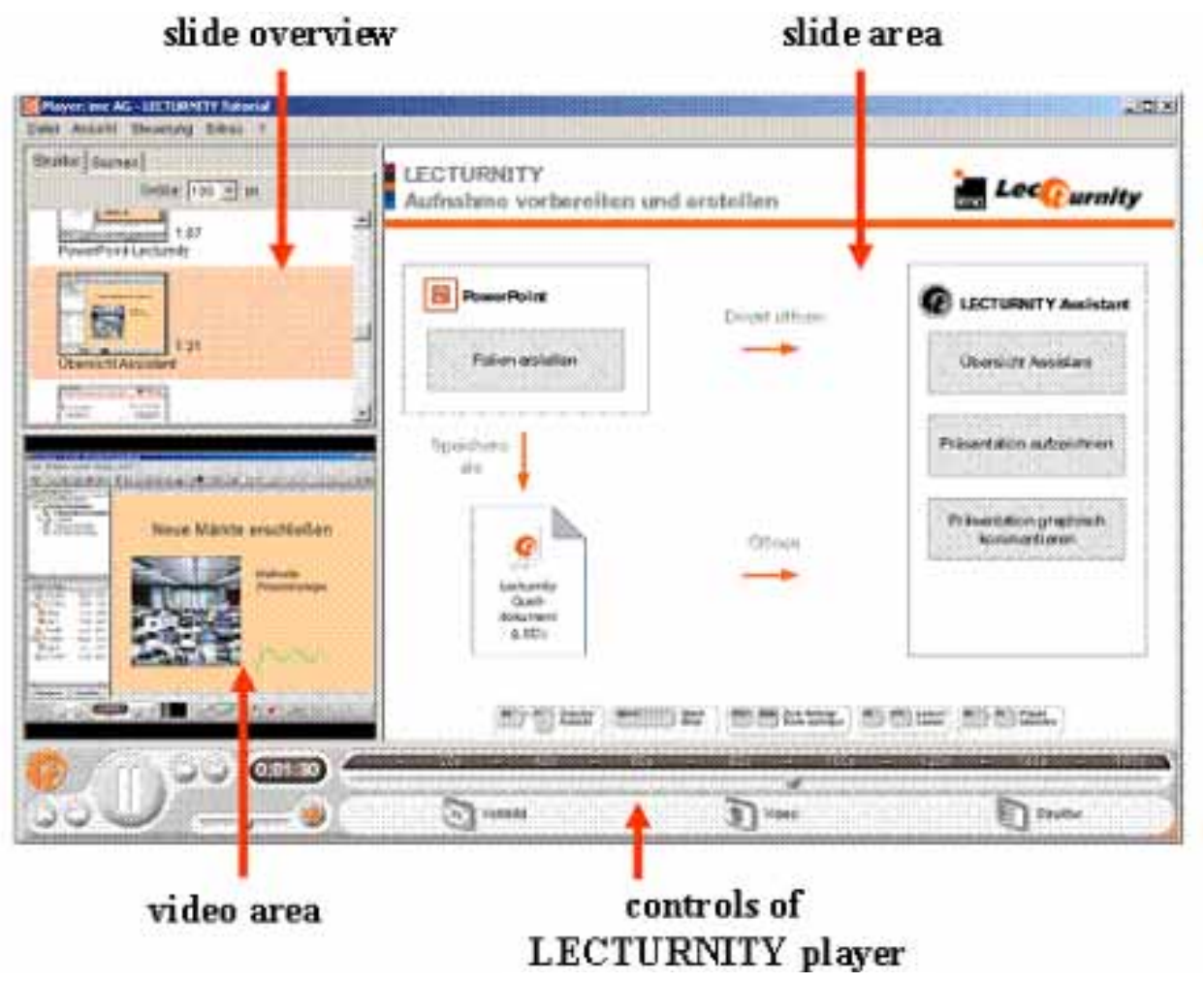

Figure 3: Screenshot LECTURNITY Player

\section{Microsoft's Producer for PowerPoint}

The Microsoft Producer (http://www.microsoft.com/office/powerpoint/producer) is a PowerPoint extension that Microsoft provides as of this writing free-of-charge. PowerPoint slides are the basic "raw material" that can be used within a Producer presentation. In addition, the software can import pre-recorded audios and videos, as well as just-in-time recordings of audio and/or video streams. Templates allow users to create presentations with a unified look-and-feel. The output is HTML and Windows media videos, with an optional XML description of the HTML files to enable a SCORM compatible learning management system (Dodds \& Schawn, 2004). However, Producer does not support the integration of meta-information or non-slide-based interactive elements within a presentation.

Figure 4 shows an example of a Producer presentation. In sum, the Producer add-on for Microsoft PowerPoint allows users to capture and synchronize audio, video, slides, and images, preview them, and publish them for viewing in Microsoft's web browser. Files produced by Producer are heavily reliant for playback on proprietary Microsoft software. While this software is free for us- 
ers of the Microsoft operating system, it is not open-source and will not display on many other browsers.

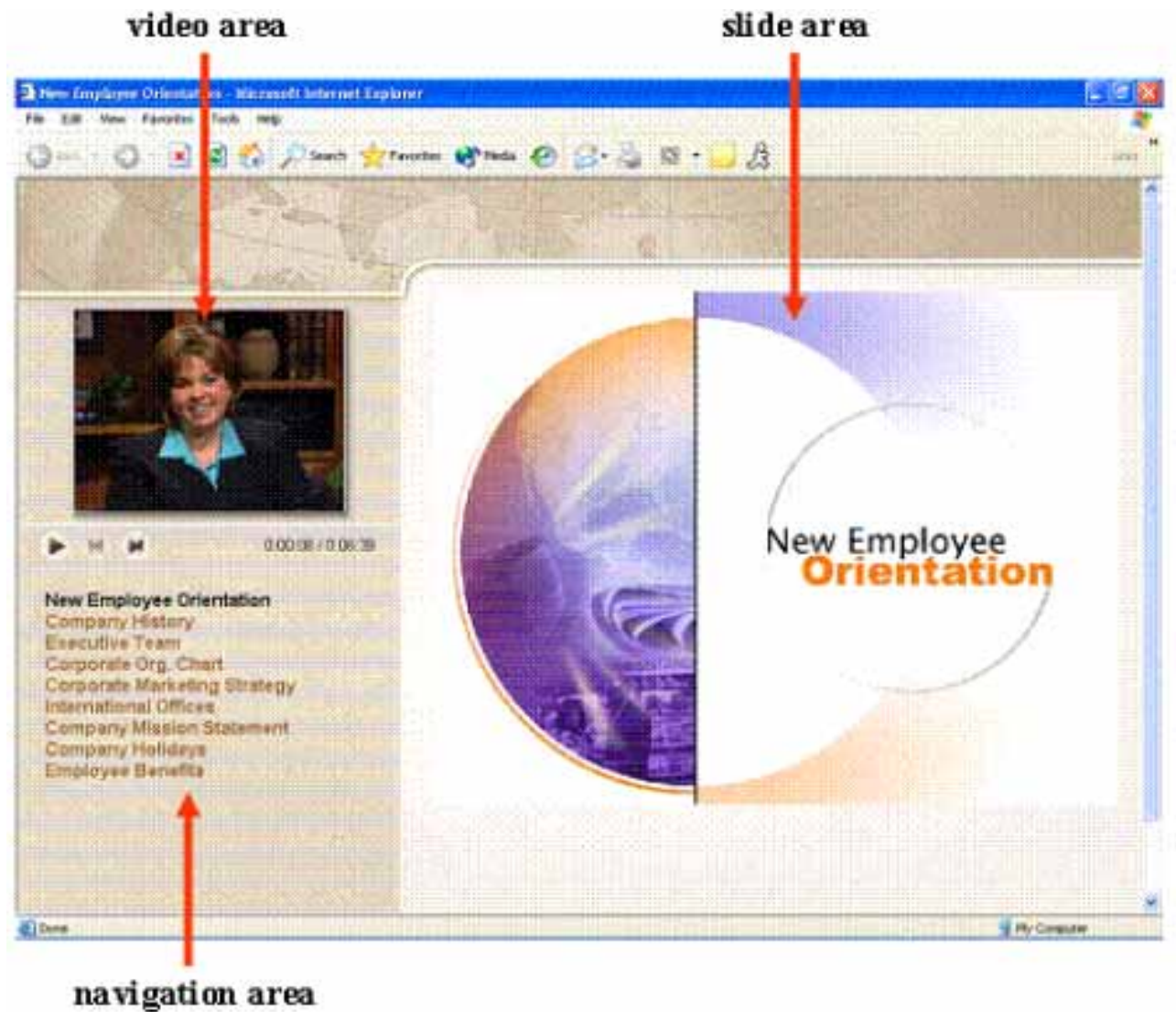

Figure 4: Screenshot Microsoft Producer Presentation

\section{I-Control}

The I-Control system (http://www.intermedia-solutions.de/produkte/index.php?s=p-icontrol) is comprised of four software modules: the capture module (to record videos), the collection module (to select and organize additional material), the director module (to compose the presentation), and a preview module. The layout for a presentation is dictated by (and limited to) a set of pre-defined templates. The user who requires a different template can either purchase one from IControl or purchase software from I-Control to create new templates.

In addition to JPG slides, PDF text, and other graphical elements, I-Control allows authors to integrate any web content within the combined area of a presentation. The Knauer (2004) review uncovered a number of software errors in I-Control. As a result, finished presentation could not playback in Netscape browsers and produced script errors in Internet Explorer. Only a video stream in Windows media format was displayed as Real Media caused further problems. Figure 5 shows a demo screenshot of the I-Control user interface in HTML format. 


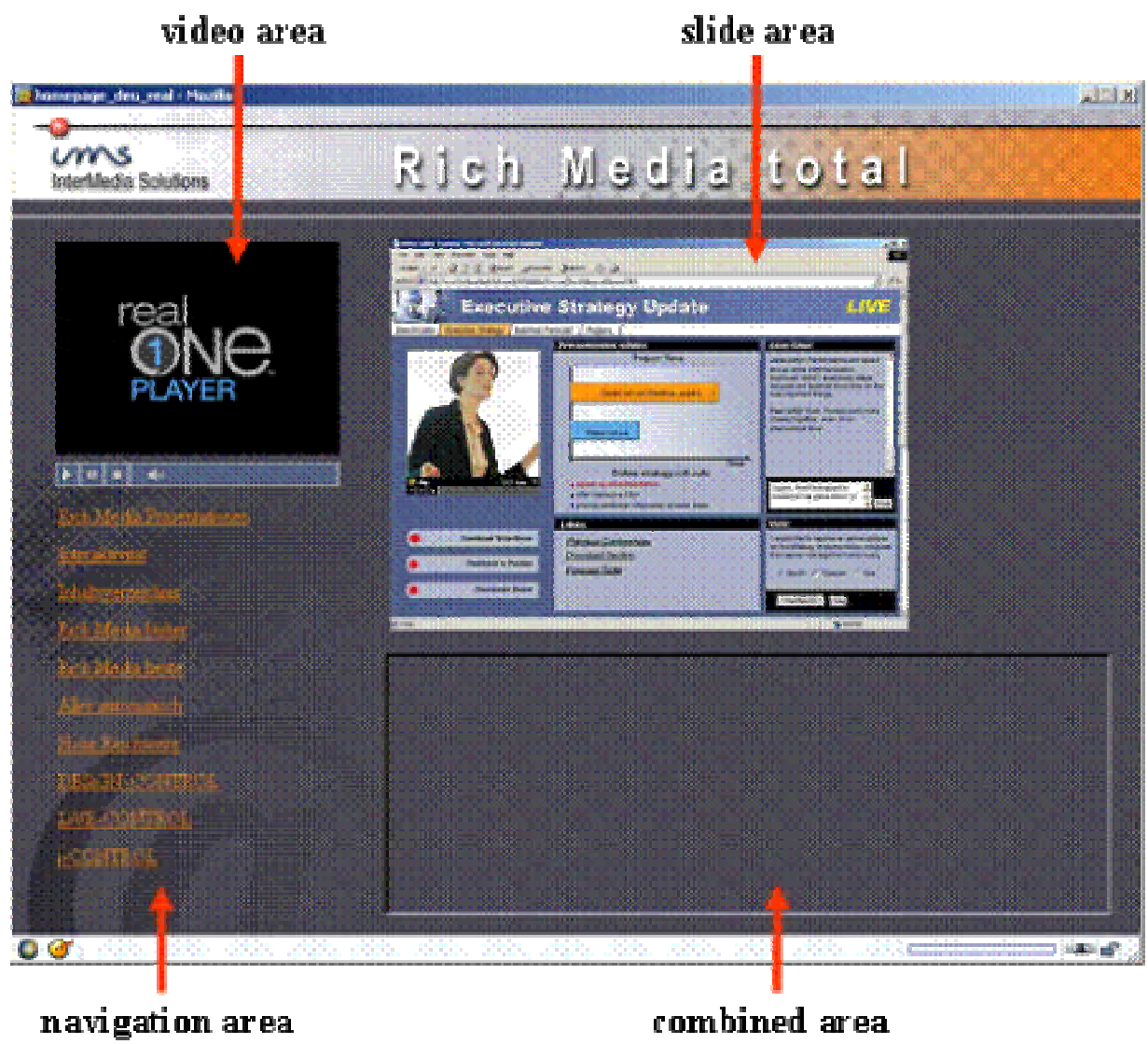

Figure 5: Screenshot I-Control Presentation

In summary, while software to produce lecturers on the web exists, the current multimedia authoring solutions have shortcomings. The following sections describe a production process and software approach for reusable web-based presentations using the LoD toolset, and show how the LoD toolset fulfills our requirements.

\section{The Steps in Creating a Presentation using the LoD Toolset}

Colleagues who use the LoD toolset to create an online presentation just follow a few, simple steps, as outlined below.

\section{Capturing Presentation Material}

The first step of the LoD authoring process is to prepare and collect the multimedia elements needed for the reusable web-based presentation. Capturing the video and audio from a talk, a discussion, or an event, requires at a minimum a single microphone and camera.

Better equipment yields better results, of course. Digital video cameras provide higher resolution and color depth. Even more important for reusable web-based multimedia presentations, though, is high quality audio. Learners do not tolerate poor audio quality in reusable web-based multimedia presentations.

Once we have captured the audio and video data from a presentation, we need to encode for web usage. A number of free encoders are available. However, the LoD system relies on the Helix Producer encoding software. This software produces output that is playable by free browser plug- 
ins (Real Player) available from Real technology and it lets us produce several different bandwidth streams at the same time. The Real Player client communicates with a Real Server application and chooses the suitable bandwidth automatically, thus relieving the user from needing to choose an appropriate bandwidth option (Bento, 2000).

Figure 6 shows the Helix Producer main window and an example of LoD encoding settings.

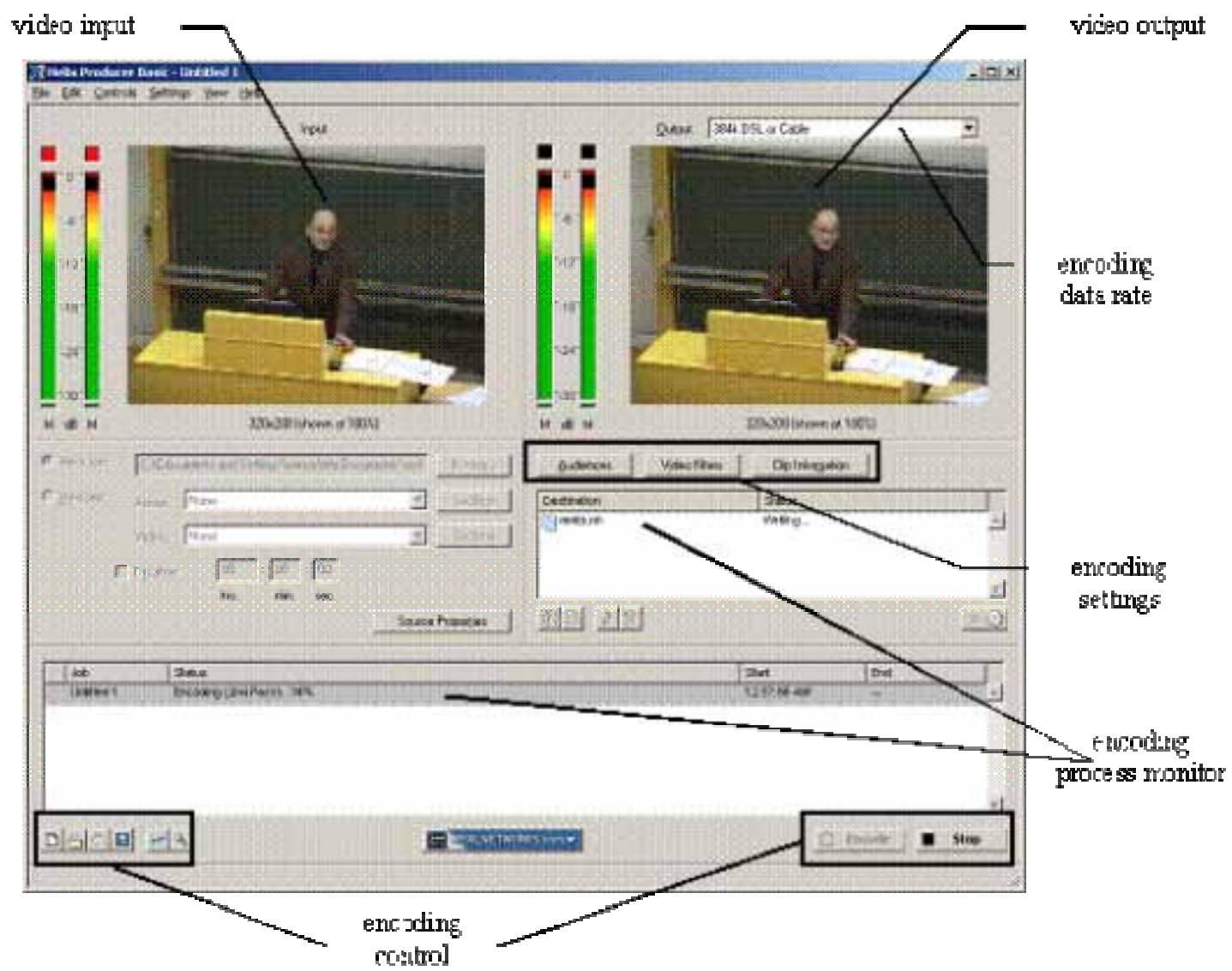

Figure 6: Screenshot Helix Producer

The Helix Producer works with any presentation software. In our case, most presenters use Microsoft PowerPoint. Consequently, in our case, PowerPoint became part of our LoD production environment. Furthermore, our LoD project team developed an add-on tool for "standard” PowerPoint that records timestamps each time the presenter moves from one slide to the next. At the end of the presentation, a timestamp list is saved for later use in the authoring process as described below.

\section{Synchronization and Navigation}

The next step in the production of a reusable web-based multimedia presentation using the LoD toolset is to consolidate all media into a single Real Video file. This requires synchronizing all multimedia material and navigation elements. Synchronized Multimedia Integration Language (SMIL) provides a practicable solution to synchronize the different media types.

Like HTML, SMIL is an easy-to-learn markup language, but while HTML's purpose is to present static pages, SMIL's purpose is to coordinate the timing of changing presentation media over the Web. It uses a single time line to coordinate and synchronize the presentation of all types of media (Michel, 2004). Figure 7 gives an example of a SMIL file: 


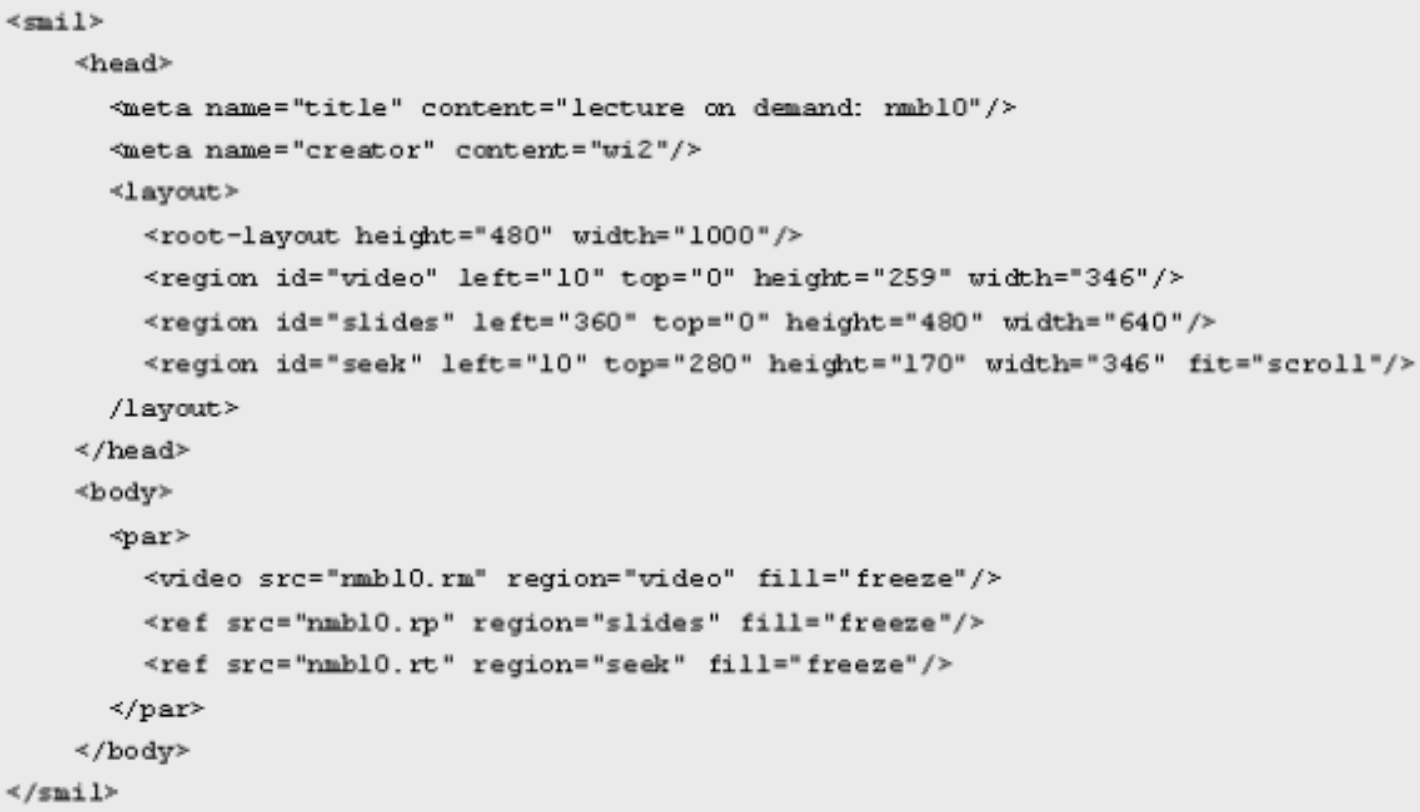

Figure 7: SMIL File Listing

A simple LoD presentation consists of the following files:

- The SMIL file to coordinate the synchronization of the presentation of the other files

- The Real Text file for navigational controls

- The Real Pix file that contains the contents of the slides

The SMIL file might also contain various meta-information about the LoD presentation, such as its title and the names of its creator. It also may define the structure and composition of the Real Player window, such as the location of regions for video, slides, subtitles, web links, or navigation elements. Note that media items can be displayed in sequence or parallel.

The Real Text file consists of a list of slide titles in combination with control commands for the Real Player. The Real Player jumps to the specified time when a user clicks on a slide title. The specified times match the ones given in the Real Pix file and as a result provide synchronization of video and slides (Real Text Authoring Guide, 2000). Figure 8 gives an example of a Real Text file.

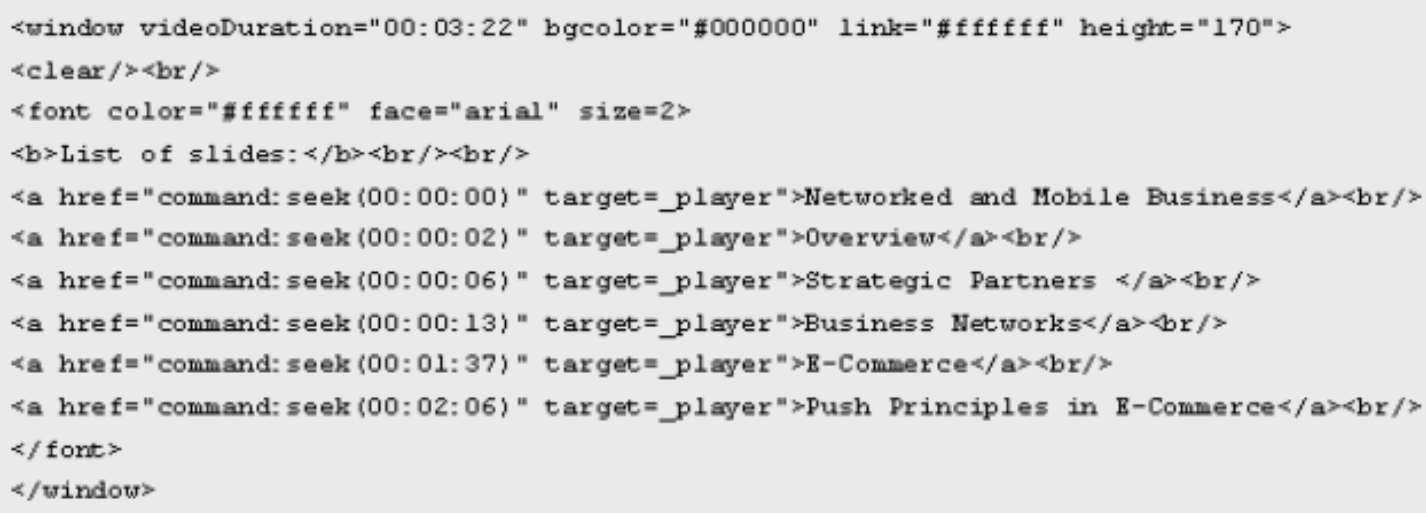

Figure 8: Real Text File Listing 
The Real Pix file contains information about the title, author, and technical details like the time format used or the duration of the presentation. Each previously produced JPEG or GIF image is put in a sequence. A number of crossover effects is used to connect the images (Real Pix Authoring Guide, 2000). Figure 9 gives an example of a Real Pix file:

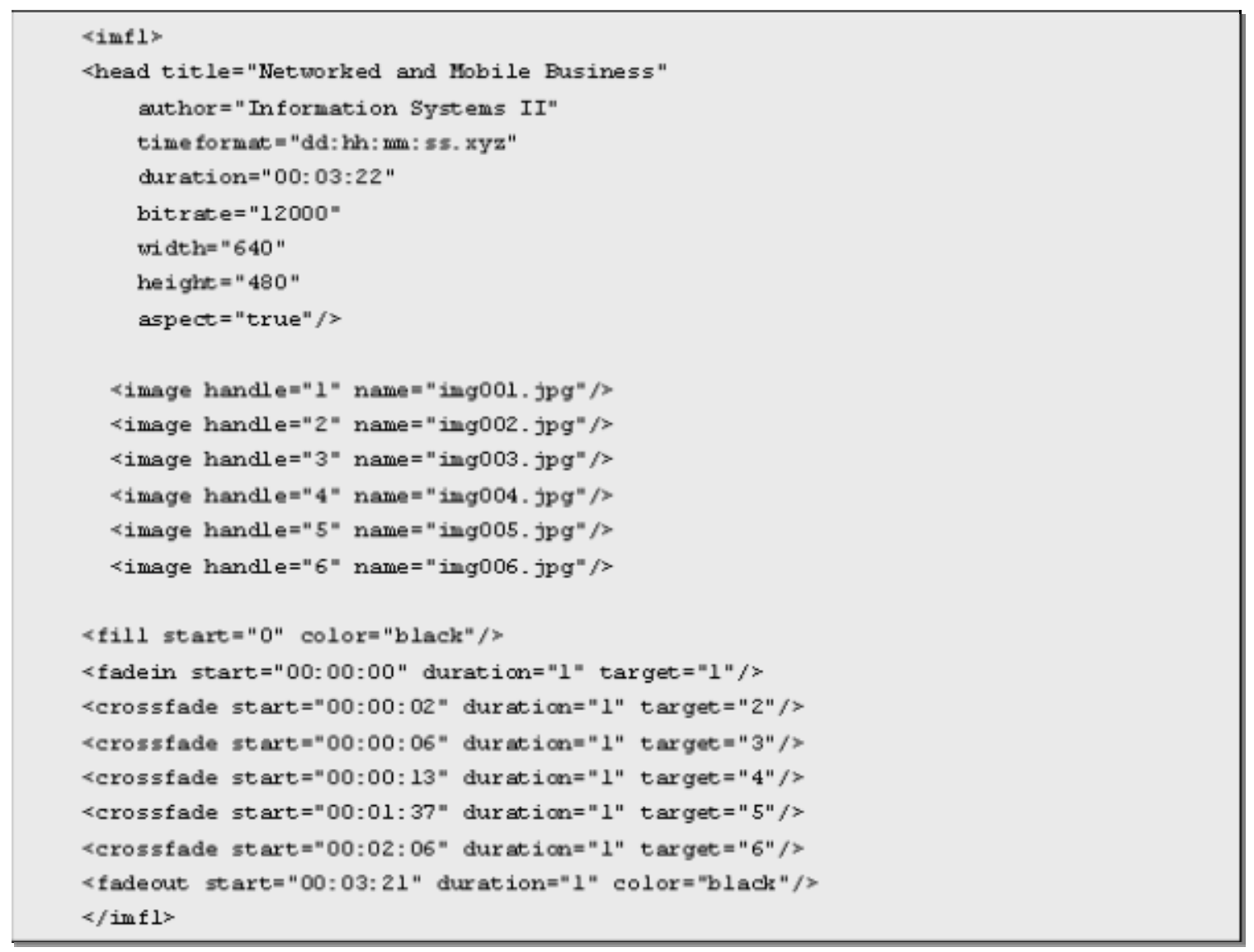

\section{Figure 9: Real Pix File Listing}

\section{Authoring and Editing}

One major problem that arises while creating SMIL-based LoD presentations is the need to edit the source code of SMIL, Real Pix or Real Text files. Although there is a variety of SMIL authoring tools on the market, none of these tools was found to be specialized in LoD production editing (Streaming Media World, 2001).

For this reason, our department developed a Java-based LoD authoring/editing tool. The tool features the following functionality:

- Import and playback of Real Video files: The tool uses a Real Media plug-in to display the video and audio data. The author can adjust the size of the video window, start, stop, and pause the video or jump to any place using a time slider.

- Import of web-compatible additional multimedia material: Every LoD presentation can contain several additional elements, as long as their format is WWW or Real Player compatible.

- Import of timestamp lists: As mentioned above, a special extension of PowerPoint creates lists of display times and the corresponding slide titles that are used to synchronize the 
different media elements of a LoD presentation. If required, the author can use the tool to re-adjust and fine tune all image display times.

- Multimedia repository for presentation data: All presentation elements are stored in a repository system with metadata support.

- On-the-fly synchronization of video and additional material: If no timestamp list is available, the authoring tool can synchronize the media elements while the video is playing.

- Organization of slides in chapters: The tool organizes slides into chapters to improve navigation. Each chapter can be broken down into as many subchapters as the author wants to.

- Integration of subtitles: The tool enables authors to add subtitles to a LoD presentation. The subtitles may be in various languages, as well. The SMIL technology ensures that the system displays the appropriate subtitle according to the language setting of the user's Real Player.

Figure 10 shows a SMIL file listing with the necessary command lines. In this listing, the root layout is expanded by the region "subtitle", which is placed under the video region. Within the body of the SMIL file, the switch instruction causes the Real Player to select either the German subtitle file (subtitles_de.rt) or the English subtitle file (subtitles_en.rt) depending on the software settings of the player.

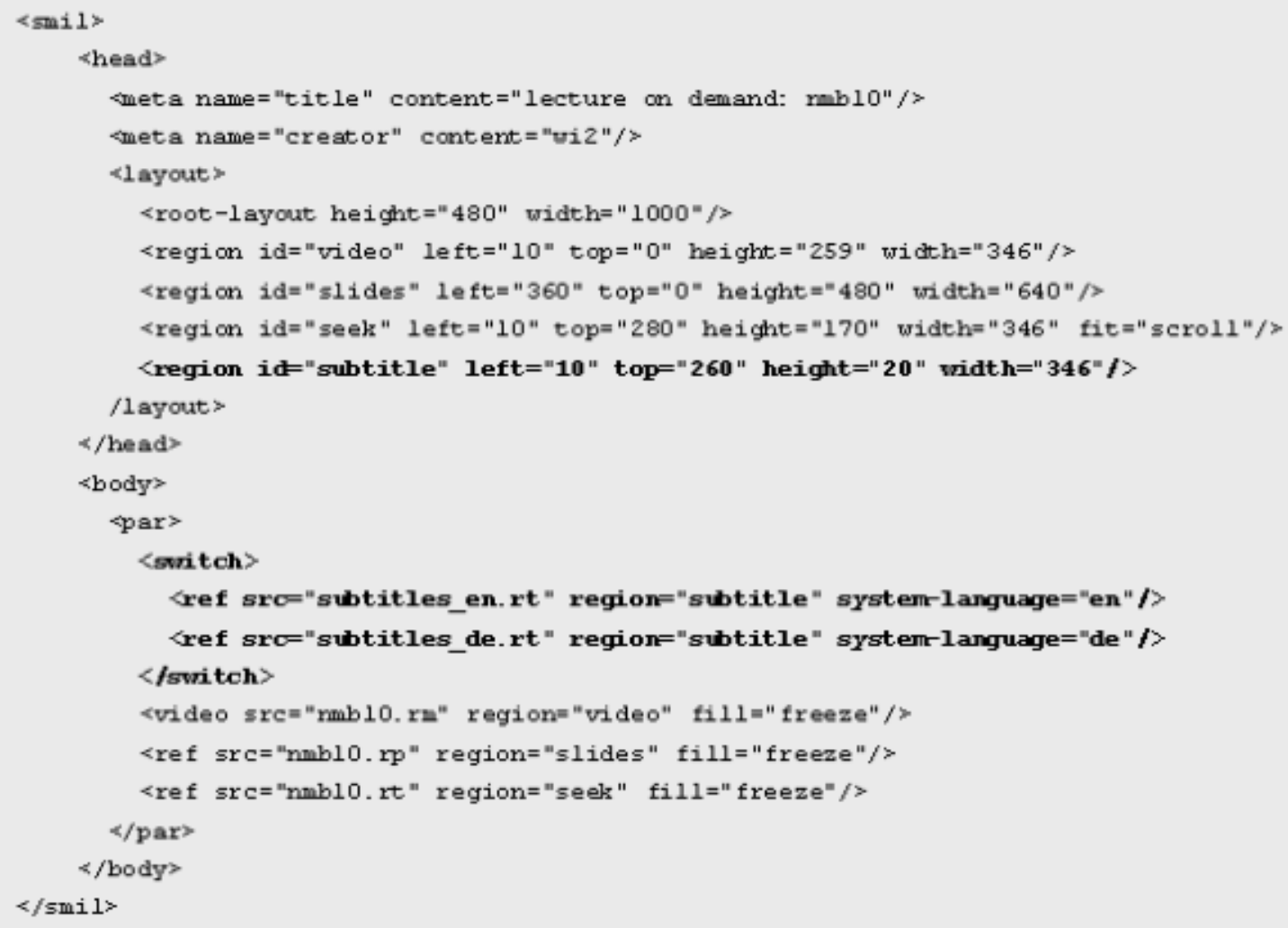

Figure 10: SMIL File Listing including Subtitles

Figure 11 provides an example of a subtitle Real Text file. Every subtitle is shown for each specific period, after which it is replaced by the next subtitle. 


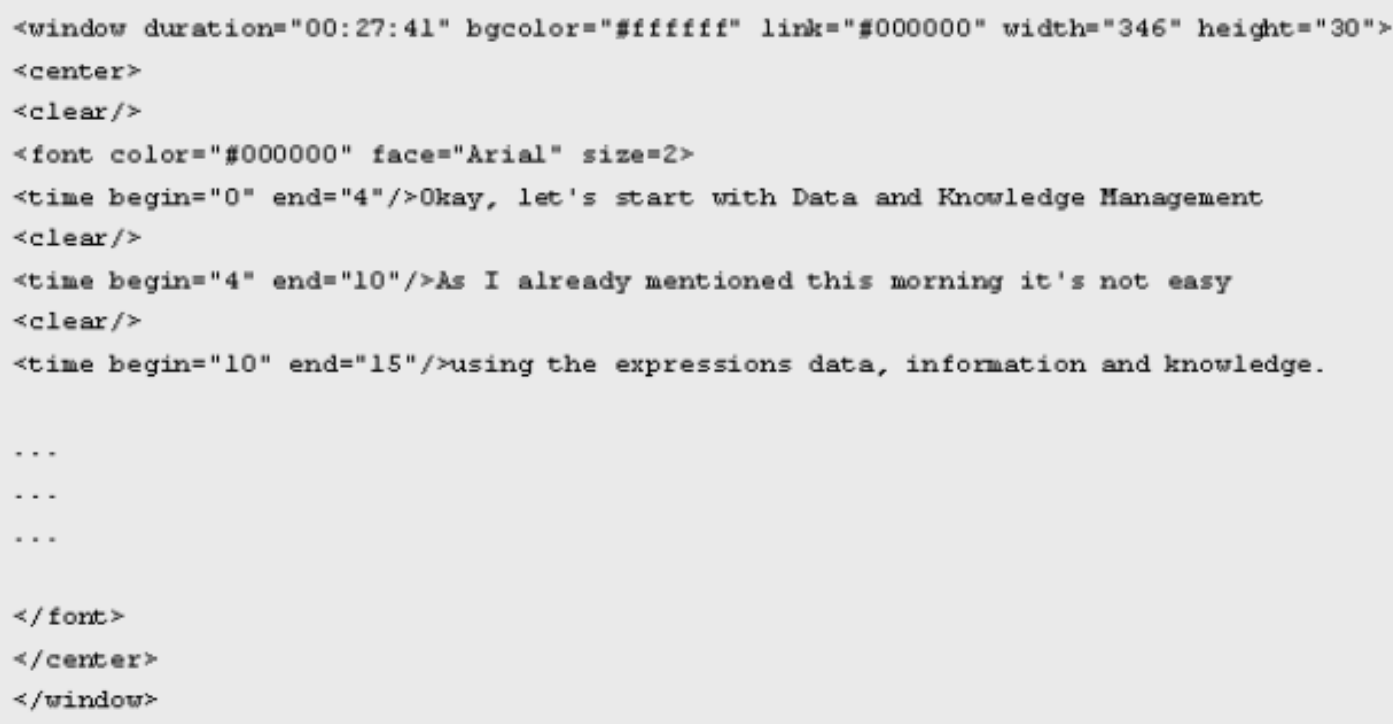

\section{Figure 11: Real Text File Listing for Subtitles}

- Integration of web link collections: The tool also allows authors to display web links within presentations and have them displayed in a particular area of the Real Player window. When a user clicks a web link, the web browser opens the corresponding website a separate window, so as not to interfere with the LoD presentation.

Figure 12 shows the modified SMIL file listing.

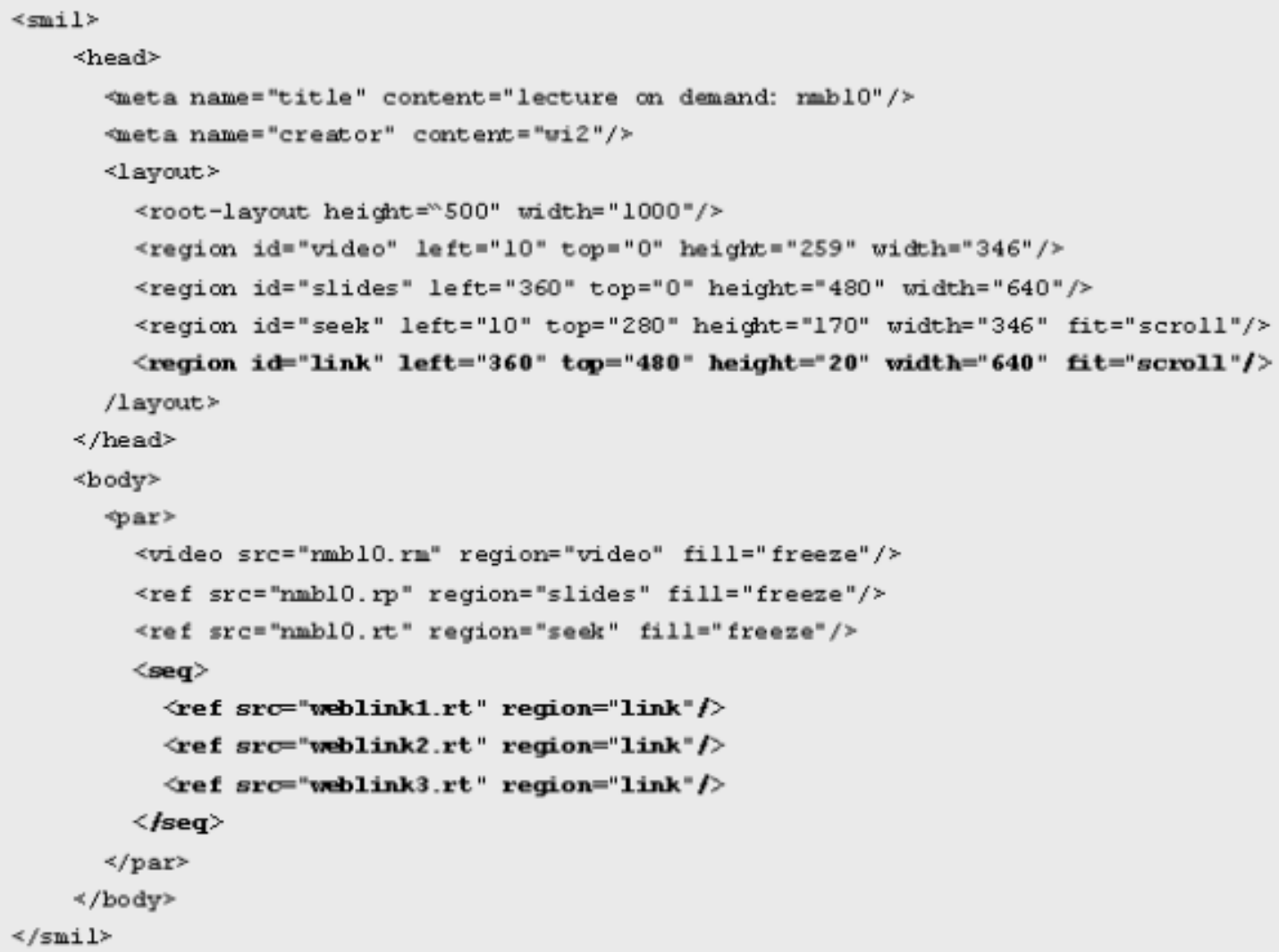

Figure 12: SMIL File Listing including Web Link Collections 
Similar to the modifications of a SMIL file with subtitles, this listing includes a new region in the root layout called "link" that is placed beneath the slides region. The body of the SMIL file contains a sequence of Real Text files. In this way, the author can change the content of the web link collection during a LoD presentation. Figure 13 gives examples of web links displayed within a LoD presentation for approximately 16 minutes. After that, the system shows the user another web link collection.

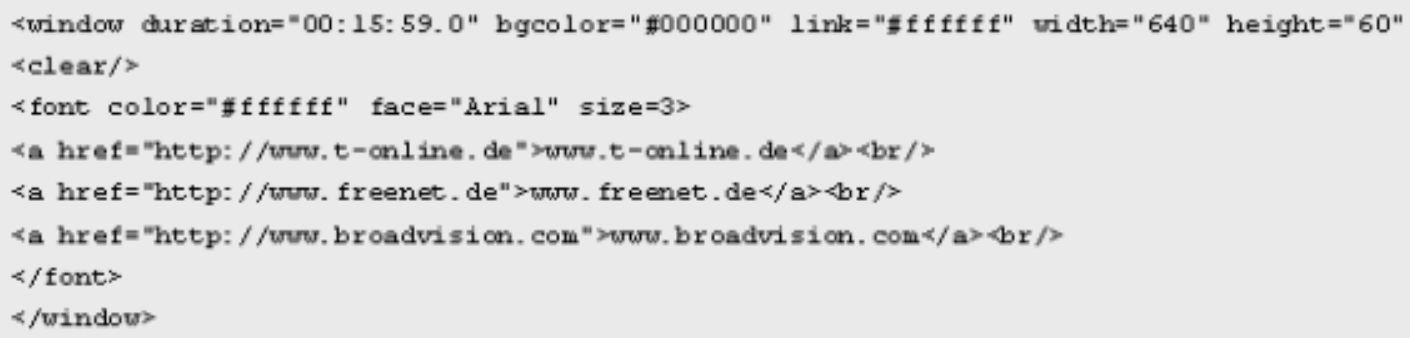

Figure 13: Real Text File Listing for a Web Link Collection

- Integration of metadata: Authors can enter any meta data they want, using the tool. Usually they will add common attributes like author name, the title of the LoD presentation and author institution or company name. In addition, they also can include metadata types from international standards like the Dublin Core or the LOM (Learning Objects Metadata) standard.

Figure 14 shows the listing of a SMIL file including Dublin Core standard metadata.

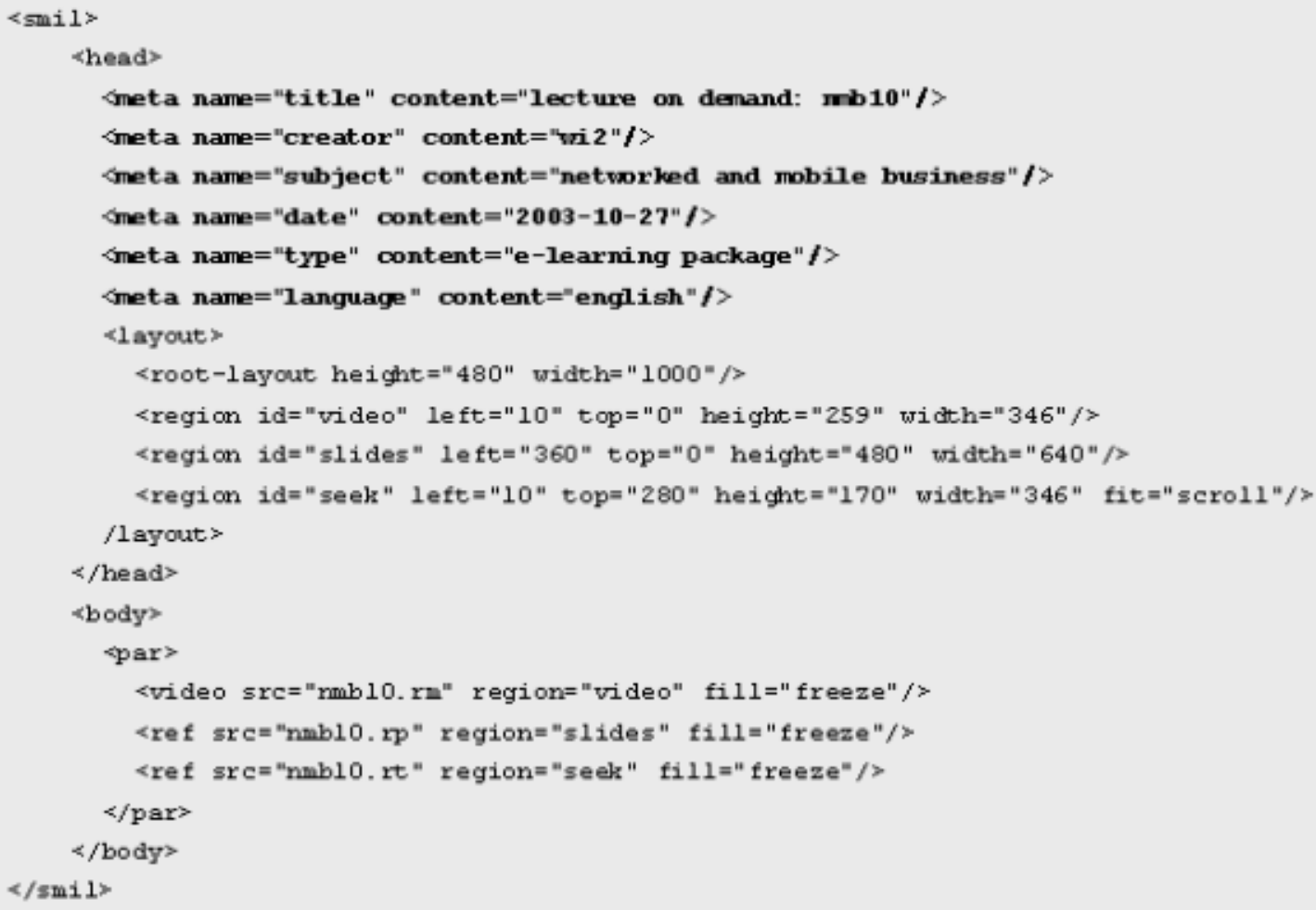

Figure 14: SMIL File Listing including exemplary Dublin Core Meta Data

- Automatic Zip-compression and transmission to web-server: The tool has a built-in compression tool that automatically compresses a presentation in a Zip-compatible package. 
- Storage of project data in XML format: The data of a current project status are saved in XML format. This format guarantees a high degree of convertibility.

Figure 15 shows a screenshot of the LoD authoring tool.

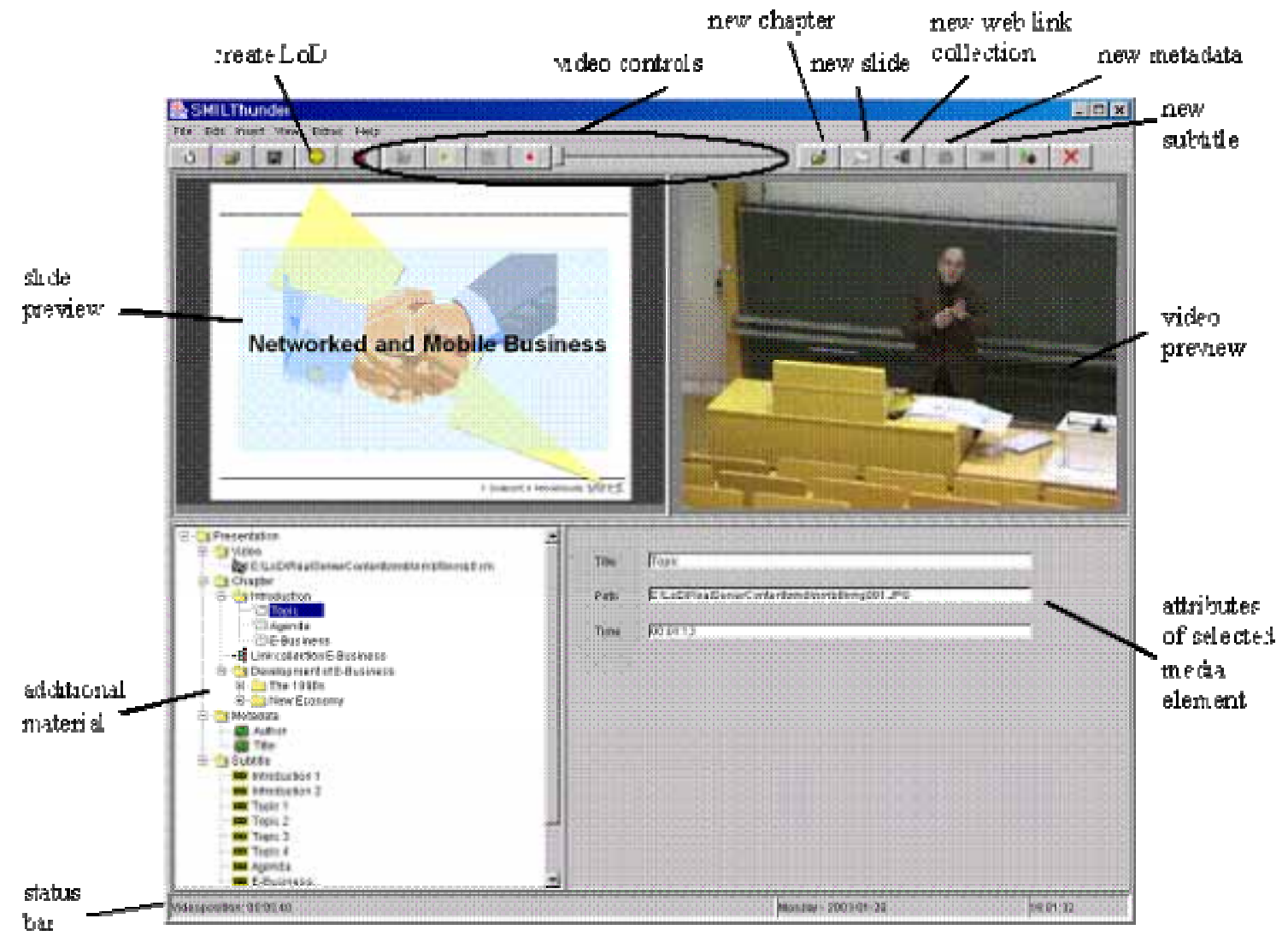

Figure 15: Screenshot LoD Authoring Tool

The output of the LoD authoring tool consists of the three files mentioned above: the SMIL file, the Real Pix file, and the Real Text file. In addition, the LoD tool creates any special files needed for subtitles and web link collections.

\section{User Interface}

To play a LoD presentation, the student can use any browser that has the RealPlayer add-in installed. Figure 16 shows a LoD presentation as might be seen by a user.

All media objects of the LoD apart from the video are stored in a multimedia repository. The video itself is streamed from the Helix server at runtime. The other elements of the LoD are retrieved from the multimedia repository following the SMIL, Real Pix and Real Text files commands.

A user who wants to benefit from the capabilities of the LoD system chooses an information package from a special web site. In order to maintain a proper overview of the content all presentations should be modularized and the single videos should not be to long. 


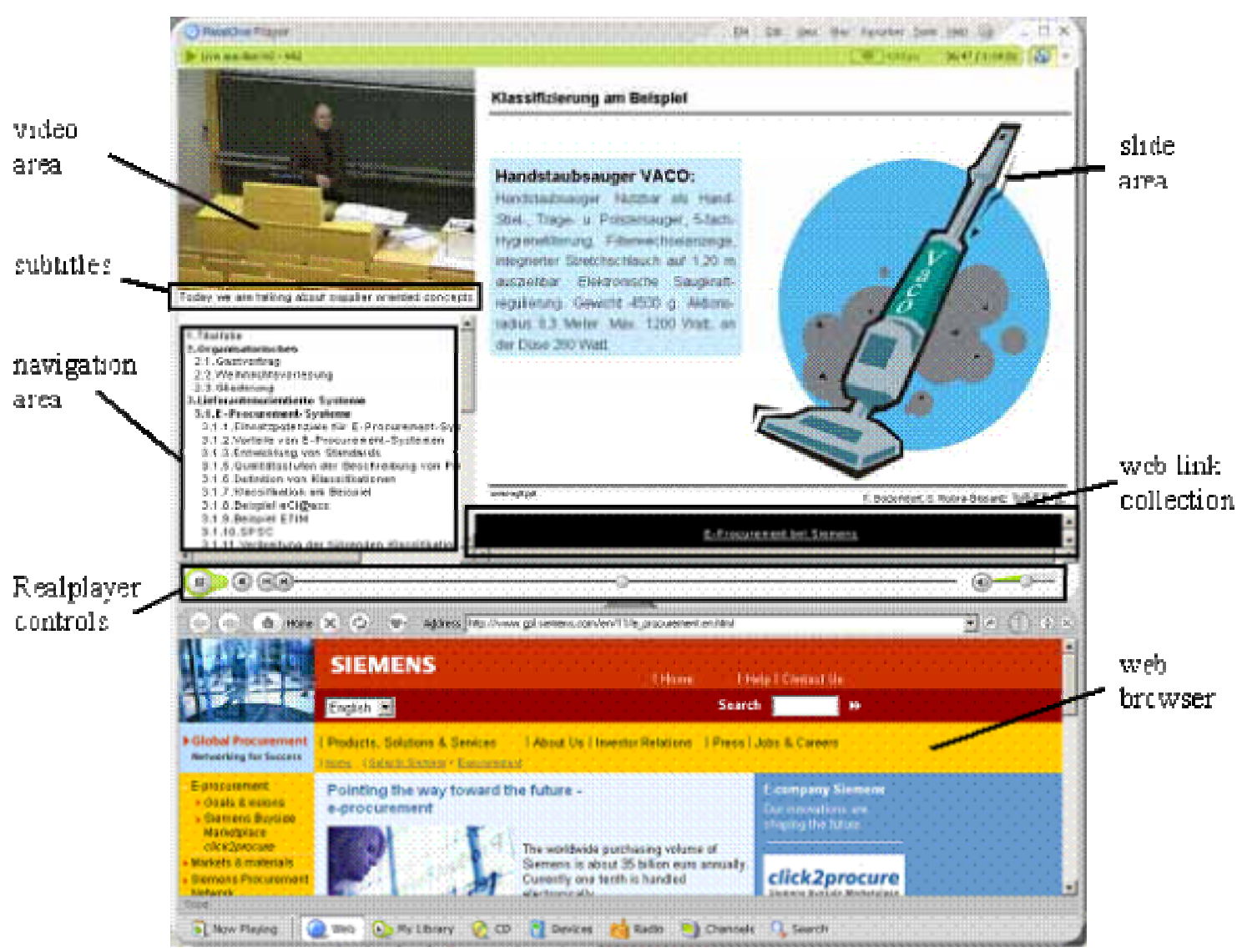

Figure 16: Screenshot SMIL Based LoD Presentation

Within the LoD presentation, the viewers can access the build-in Real Player navigation elements as well as the special LoD navigation. The former allows jumping to any position of the presentation using the time slider, the latter makes it possible to exactly pick one presentation slide and see and hear the corresponding part of the information package.

The video stream remains synchronized with the additional material. When a user selects a web link, the build-in browser of the Real Player or the standard browser of the used computer displays the corresponding material.

Web links can serve a variety of purposes in a presentation. First, web resources like company or university websites, scientific articles or search engines for own research might help users understand the information given in the LoD presentation better. Second, the system allows us to link previously prepared background information, such as additional text material, to the LoD presentation in HTML or PDF format. Indeed, the web link to a text source can either provide the entire text or point to the exact text passage the LoD presentation is currently dealing with.

Third, we can insert web links in a LoD presentation to carry out online or offline assessments. We ask users to answer several questions on the content presented in the LoD. The system then either corrects these answers immediately or posts them to a tutor, who manually marks the tests and provides users with individual feedback. 


\section{Conclusion}

Going virtually with at least a certain range of web-based information offerings is not a choice any more for most academic education institutions. Even if it is not a university's professed aim to become a "virtual university" with all consequences, a growing demand for off schedule offerings has to be satisfied. Exorbitant costs for sophisticated multimedia productions for reuse often discourage staff members to simply give it a go and venture a serious attempt to put some traditional courses on the Internet as well.

The solution proposed in this paper is a low cost, fast and practical approach to ensure a quick supply of quality controlled reusable web-based multimedia presentations. Any presenter or instructor who carries out presentations in the traditional way is able to perform the LoD authoring process at minimum additional expenses (Schertler \& Bodendorf, 2001).

At our department, we record and transform every single lecture into a LoD presentation. We offer these video lectures on our website both for online streaming and for download. In addition, we provide users without the Internet access with an offline version on CD-ROM.

Our students mainly use the LoD presentations for recap and as a preparation tool for the exams.

Readers interested in our tool can contact us via e-mail. Our department should accompany the installation. We provide additional information on using the LoD toolset at

(rtsp://131.188.219.155:554/demo/start.smil). Readers can view a demo presentation produced with the LoD toolset at (rtsp://131.188.219.155:554/ebec/ebec01/ebec01.smil)

\section{References}

Bento, A. (2000). Developing a class session using audio and video streaming. In A. Aggarwal (Ed.), Webbased learning and teaching technologies: Opportunities and challenges (pp. 103-116). Hershey, PA: Idea Group.

Dodds, P. \& Schawn, E. T. (2004). SCORM $20042^{\text {nd }}$ Edition Overview. Retrieved 27 April 2005 from http://www.adlnet.org/screens/shares/dsp_displayfile.cfm?fileid=992

Klein, A. (1999). ATM-based infrastructure for teleteaching at university. 2nd International Conference on ATM, Colmar.

Knauer, A. (2004). Entwicklung eines internetbasierten E-Lecture-Autorensystems auf Basis eines multimedialen Lehrmittelrepositories. Diploma thesis, Department of Information Systems, Nuremberg.

Michel, T. (2004). Synchronized multimedia. Retrieved 29 September 2004 from http://www.w3.org/AudioVideo

Real Pix Authoring Guide. (2000). Retrieved 29 September 2004 from http://service.real.com/help/library/guides/realpix/realpix.htm

Real Text Authoring Guide. (2000). Retrieved 29 September 2004 from http://service.real.com/help/library/guides/realtext/realtext.htm

Schertler, M., \& Bodendorf, F. (2001). Teacher guidance to digital lectures. ED-MEDIA 2001, Association for the Advancement of Computing in Education, Tampere.

Streaming Media World. (2001). Retrieved 29 September 2004 from

http://smw.internet.com/smil/tools/authoring.html 


\section{Biographies}

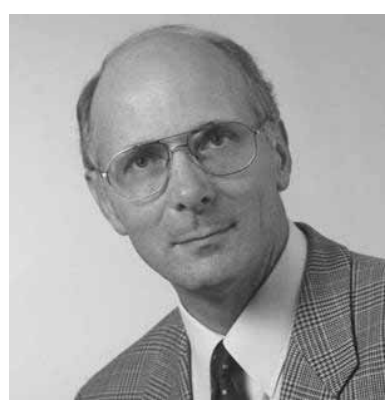

Freimut Bodendorf is head of the Department of Information Systems at the University of Erlangen-Nuremberg. He is a member of the executive committee for media-based education at the University of Erlangen-Nuremberg. He also serves as a member of the advisory council of the Virtual University of Bavaria.

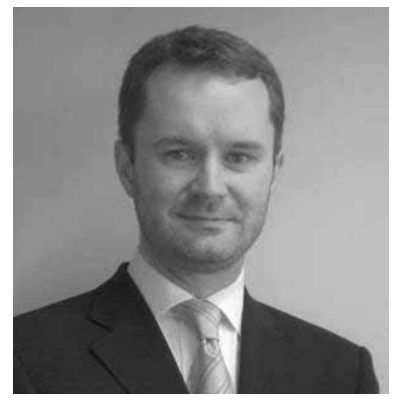

Manfred Schertler is a research assistant in the Department of Information Systems at the University of Erlangen-Nuremberg. His research activities focus on knowledge management, e-learning applications, and teacher support in distributed learning environments.

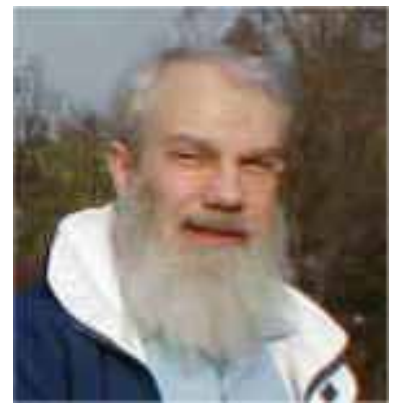

Eli Cohen is a director of the Informing Science Institute and professor at the Kozminski School of Entrepreneurship and Management in Warsaw. He also organizes the Informing Science + Information Technology joint conference. 